\title{
Les villes redécouvrent l'eau
}

\author{
Gilles Hubert \\ Cergrene, La Courtine - 93167 Noisy-le-Grand
}
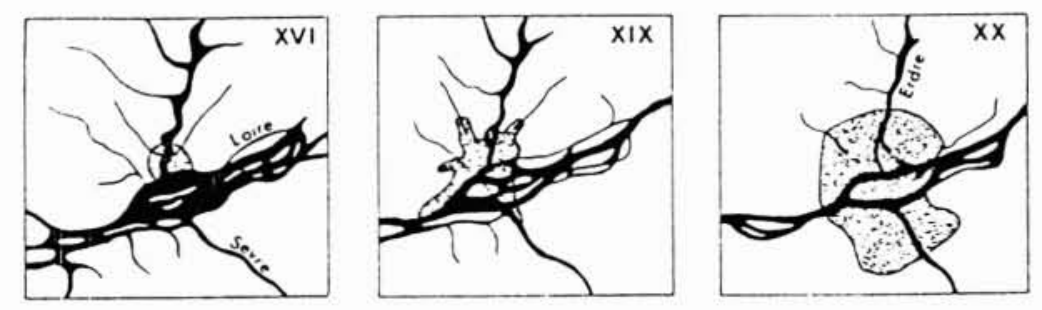

\section{Le poids de l'histoire}

L'eau a toujours été un facteur essentiel dans l'organisation et le fonctionnement des sociétés humaines.

Historiquement, ce sont les rivières au régime lent et régulier qui ont servi de point d'accroche aux cités. En devenant le principal moyen d'échange et de transport de marchandises mais aussi une matière première pour l'artisanat et les manufactures, elles ont participé très largement au développement et à la prospérité des villes.

On peut citer le cas de la ville médiévale qui doit en grande partie son essor à la présence de l'eau. La constitution d'un important réseau de canaux intra-muros et extra-muros, entre le $x^{c}$ et le XIII ${ }^{c}$ siècle, permet de fournir en permanence aux métiers de l'eau (tanneurs, régissiers, drapiers, teinturiers...) une eau vive et abondante. On voit même s'esquisser tout au long de ce réseau une hiérarchie des activités en fonction des besoins qualitatifs et quantitatifs propres à chacun ; ce qui permet de régler en partie les conflits d'usage.

Pendant des siècles, les relations étroites qui s'établissent entre la rivière et la ville semblent indissociables. Pourtant la révolution industrielle du $\mathrm{XIX}^{\mathrm{e}}$ siècle va bouleverser ces rapports, considérés par beaucoup comme étant « harmonieux ". Les progrès techniques en matière d'hydraulique ainsi que la réalisation des réseaux d'alimentation en eau potable et d'assainissement ne sont pas sans influence sur la place de l'eau dans la ville.

Dès 1850 la rupture s'amorce. Elle s'amplifie au fur et à mesure que l'urbanisation progresse. Le point d'orgue est atteint après la seconde guerre mondiale au moment de la reconstitution. La croissance urbaine est sans précédent et elle se fait largement dans l'ignorance au cycle de l'eau.

Le schéma ci-dessus, qui montre la régression du réseau hydrographique de la ville de Nantes, entre le $\mathrm{XvI}^{\mathrm{e}}$ et le $\mathrm{xx}^{\mathrm{e}}$ siècle, en fonction de son développement, est tout à fait représentatif de cette stratégie de l'oubli.
Le divorce sera à peine prononcé et déjà de nombreux dysfonctionnements apparaîtront. Les problèmes rencontrés par certaines villes pour assurer correctement leur assainissement ou leur alimentation en eau potable, l'augmentation de la fréquence et de l'intensité des inondations, ou encore l'aggravation des phénomènes de pollution imposent rapidement une nouvelle dimension à la gestion de l'eau en général et des rivières urbaines en particulier. Parallèlement à cela, la sensibilisation de l'opinion publique, aux aspects écologiques entraîne une remise en cause des logiques d'aménagement trop sectorielles et trop techniciennes qui ignorent les principes d'équilibre, de régulation et de cycle de l'eau. Enfin, l'accroissement des demandes de loisirs et d'espaces verts de proximité émanant des citadins, comme le désir d'enracinement local et d'appropriation de l'espace conduisent l'ensemble des acteurs qui interviennent dans la gestion de l'eau à porter un nouveau regard sur l'aménagement des cours d'eau et de leurs espaces adjacents.

\section{Le second souffle}

A partir des années 1970 , les collectivités locales vont être de plus en plus nombreuses à lancer des opérations d'aménagement de rivière et à redonner une place de choix à l'eau dans l'espace urbain. Indiscutablement, les problèmes techniques de gestion de l'eau (contrôle des flux, lutte contre la pollution...) jouent un rôle de catalyseur pour passer à l'action. Toutefois, d'autres arguments sont également avancés et dans certains cas ils prennent le pas sur ces questions.

On assiste en quelque sorte à un renouvellement des approches d'aménagement ; des efforts d'imagination sont déployés pour apporter des réponses plus globales. 


\section{G. HUBERT}

La mise en valeur d'un cours d'eau urbain devient source de nombreux enjeux, nous ne retiendrons ici que les principaux :

- Se servir de l'eau pour promouvoir le milieu urbain et/ou changer l'image de marque d'un quartier.

- Faire de l'eau un élément fort de l'aménagement de l'espace en l'utilisant comme composant principal d'une trame urbaine.

- Mettre en valeur le patrimoine architectural, culturel et historique d'une ville en réhabilitant la place de l'eau.

- Réintroduire la nature en ville en intégrant la dimension écologique d'un cours d'eau à son aménagement.

- Créer une nouvelle sociabilité urbaine en utilisant la rivière et les berges comme un support des pratiques de loisirs, comme des lieux de convivialité et des espaces collectifs.

Les points de synergie qui peuvent apparaître entre le système aquatique et le milieu urbain au moment d'une opération d'aménagement ne doivent pas faire oublier les contraintes de toute nature dont il faut s'affranchir ou qu'il faut assimiler pour faire avancer le projet, assurer ensuite sa mise en œuvre, puis pérenniser l'aménagement.

\section{Les obstacles à surmonter}

L'aménagement d'une rivière, même lorsque l'on se contente d'agir sur un tronçon donné, met en avant l'aspect spatio-temporel de la gestion de l'eau (relations amont-aval, rapports rivières-nappes, interactions entre les éléments du cycle de l'eau et aménagement du territoire, effets induits d'une action sur l'utilisation future de la ressource...). Face à la complexité des milieux aquatiques, on apporte généralement des réponses fondées sur des critères de simplification du système. Ne serait-ce que pour des raisons d'efficacité technico-économique des actions d'acceptation sociale des opérations et de préservation du patrimoine il devient toutefois nécessaire de rompre avec les approches d'aménagement trop sectorielles pour préférer les démarches dites "intégrées et globales".

Si la plupart des acteurs qui interviennent dans le domaine de l'eau tiennent aujourd'hui ce discours, le passage à l'acte reste difficile et cela pour plusieurs raisons.

Certains invoquent la complexité des mécanismes en jeu, les difficultés techniques rencontrées et la diversité des usages à prendre en compte. D'autres se retranchent derrière les complications institutionnelles et juridiques ou la multiplicité des acteurs.

En réalité, ces hésitations traduisent chez les décideurs et les techniciens une résistance au changement et un manque réel de savoir-faire en matière d'approche globale. Partant de cette hypothèse, les recherches que l'on peut mener pour tenter de débloquer la situation ou du moins y participer doivent s'intéresser aux processus de décision qui sont liés au montage d'un projet et proposer des démarches méthodologiques ainsi que des outils d'aide à la décision spécifiques.

\section{La décision en question}

Une opération d'aménagement de rivière, action transversale par excellence, consiste en quelque sorte à instaurer un nouvel état du système qui est capable de :

- répondre à la fois aux différents problèmes rencontrés, aux demandes des usagers, à la pluralité des points de vue et aux potentialités du milieu;

- s'inscrire dans un cadre géographique significatif par rapport aux réalités du cycle de l'eau et dans un contexte juridico-institutionnel donné.

La poursuite de ces objectifs nécessite en préalable de développer la connaissance de l'hydrosystème, avant d'agir. Dans un deuxième temps, elle peut conduire à la mise en œuvre d'actions en termes d'aménagement et de gestion du milieu aquatique et/ou terrestre mais aussi à faire des choix entre usage ou définir des règles limitant leur pratique. Elle peut également rechercher une modification du comportement de certains acteurs (usagers directs ou indirects). Elle peut enfin se traduire par la création de structures spécifiques d'études, d'aménagement et de gestion ou bien encore par l'adaptation de structures existantes pour une prise en charge du projet.

On se trouve dans une situation où les notions de processus décisionnel et de prise de décision sont très prégnantes. Il apparaît que la préparation de la décision est aussi importante que la décision elle-même. En s'inspirant des travaux de J.L. le Moigne sur la modélisation des activités de conception et de décision au sein des organisations, on peut formaliser ces aspects de la manière suivante:

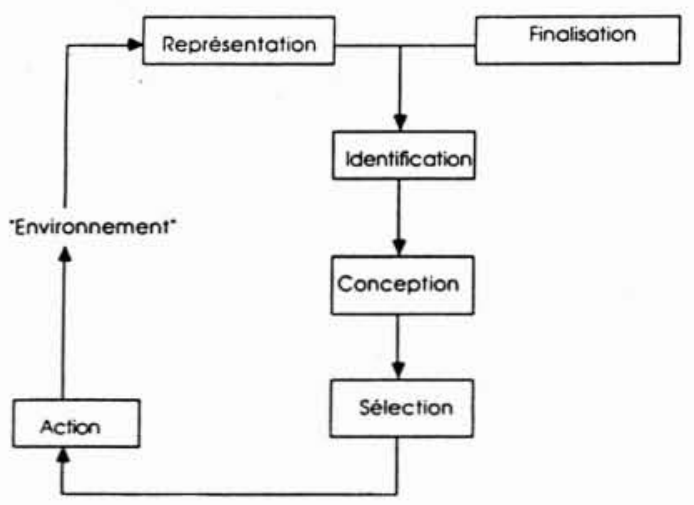

- La phase de représentation du système correspond à l'activité de recueil et de traitement des données nécessaires pour établir une image de site.

- La phase de finalisation a pour objet de mettre en avant les objectifs à atteindre, c'est-à-dire, les usages et les fonctions à favoriser ou à permettre.

- La phase d'identification issue du croisement des deux étapes précédentes, a trait à la définition des problèmes rencontrés et des objectifs envisageables mais aussi à la mise en évidence des besoins d'informations complémentaires. 
- La phase de conception correspond à la recherche des solutions qui permettent d'atteindre totalement ou partiellement les objectifs fixés.

- La phase de sélection conduit au choix d'une solution parmi l'ensemble.

- Enfin, la phase d'action consiste à mettre en œuvre les opérations choisies donc à modifier l'environnement.

La forme définitive d'un projet est donc le résultat d'un long processus itératif qui est jalonné d'un certain nombre de décisions intermédiaires prises à la lumière des constatations faites au cours du recueil des données sur le site. C'est l'ensemble de ces "micro-décisions" qui va donner la décision finale.

Le processus de décision qui vient d'être décrit nous amène à poser trois types de questions :

- Comment réaliser un diagnostic aussi complet que possible du système étudié et surtout comment agencer les connaissances multiples pour connaître les potentialités au milieu?

- Quelles sont les structures institutionnelles les mieux à même de mener à bien ce type d'approche ?

- Quelles sont les procédures juridiques les mieux adaptées pour soutenir une telle démarche?

Nous ne traiterons pas de la première question dans le cadre de cet article. Nous aborderons seulement les deux questions suivantes.

\section{L'organisation des acteurs}

Face à la grande diversité des acteurs et donc des points de vue à considérer simultanément dans le cadre d'une approche globale, il apparaît nécessaire de se pencher sur les modes d'organisation possibles.

Il est difficile de rendre compte de la réalité du système d'acteurs concerné directement ou indirectement par un aménagement de rivière, particulièrement en site urbain où le nombre et la diversité des intervenants sont importants.

En considérant tout d'abord l'étape de réflexion, au cours de laquelle sont définis les objectifs d'action et sont choisies les solutions, nous sommes principalement en présence des groupes d'acteurs suivants :

- les élus des collectivités territoriales qui sont les opérateurs essentiels de l'aménagement des rivières, ils constituent le pôle de décision ;

- le groupe d'étude (équipe pluri-disciplinaire) qui va réaliser le diagnostic, ils forment le pôle d'expertise ;

- les services de l'Etat via le préfet qui sont chargés de la gestion de la ressource en eau, ils jouent un rôle de conseil et de contrôle ;

- les usagers de l'eau qui sont les premiers concernés par les aménagements, ils peuvent former un pôle consultatif.

On peut noter que cette classification reste très théorique dans la mesure où certains acteurs peuvent jouer plusieurs rôles à la fois. Ainsi, les services extérieurs de l'Etat peuvent assurer simultanément une fonction de maîtrise d'œuvre d'étude et de contrôle (police des eaux), même s'il s'agit de deux services différents. Ils peuvent ensuite jouer le rôle de maître d'œuvre d'aménagement. De même, le groupe d'étude peut être rattaché à la collectivité (services techniques) ou être indépendant (bureau d'étude privé). Cette remarque est d'ailleurs également valable au moment de la mise en œuvre d'une opération.

La qualité du projet réside en grande partie dans la dynamique d'acteur qui peut être créée au moment de la préparation de la décision. Cette dynamique doit être fondée sur la mise en relation des acteurs pré-cités, les résultats des études (traduits dans un langage clair pour l'ensemble des personnes concernées) étant utilisés comme base de dialogue entre intervenants (communication des résultats des études, circulation des informations, participation aux décisions...).

Doit-on créer une structure spécifique pour mettre en relation ces actions ou bien utiliser les institutions existantes?

Il n'y a pas de réponse définitive à cette question car il peut exister localement des structures de réflexion capable d'organiser cette étape centrale (agences d'urbanisme, associations loi de 1901...).

L'essentiel est de réussir à "institutionnaliser » d'une manière souple et pas nécessairement définitive une expression convenable des communautés d'intérêt en présence sur le site. Il peut être intéressant de rechercher l'existence simultanée d'une structure de maîtrise d'ouvrage (d'étude et/ou d'aménagement) et d'une structure de concertation.

Concernant le $2^{\mathrm{e}}$ type de structure, on peut noter que la nouvelle loi sur l'eau du 3 janvier 1992 suggère de créer par sous-bassins des commissions locales de l'eau (CLE). Composées de trois collèges (élus des collectivités locales, représentants des usagers, administrations), les CLE pourront constituer le noyau opérationnel d'une consultation élargie, notamment au moment de l'élaboration et de la mise en œuvre des schémas d'aménagement et de gestion des eaux (SAGE).

Lorsqu'il s'agit de passer à l'action, c'est-à-dire, de mettre en œuvre la décision, il devient impératif de mettre en place ou de désigner la structure adéquate pour assurer la maîtrise d'ouvrage d'aménagement et de gestion. Il existe à cet égard différents modes d'organisation institutionnelle :

- les associations syndicales de propriétaires riverains;

- les collectivités locales et leurs groupements (districts urbains, communautés urbaines);

- les syndicats intercommunaux (à vocation unique ou multiple);

- les ententes interdépartementales ;

- les syndicats mixtes;

- les sociétés d'économie mixte.

Quelle que soit la structure choisie, il est nécessaire de lui affecter les moyens techniques, juridiques et financiers correspondants à sa mission. Parallèlement à cela, il s'avère indispensable de maintenir la concertation entre acteurs, initiée précédemment au niveau local. A cet égard, les commissions locales de l'eau pourront jouer ce rôle, dans la mesure où elles constitueront des assemblées délibérantes permanentes. 


\section{G. HUBERT}

\section{Le choix des procédures juridiques}

Il existe une grande diversité d'instruments juridiques et de nombreuses possibilités pour accompagner les temps forts d'un processus de décision et/ou pour appuyer la mise en œuvre des actions sur le terrain. Sans rechercher l'exhaustivité, ni tenter d'établir a priori un programme quelconque d'utilisation, il est possible de distinguer :

- les outils d'orientation, qui fournissent une aide précieuse pour enrichir la réflexion et concevoir un projet (les cartes d'objectifs de qualité, le schéma de vocation piscicole, les schémas d'aménagement et de gestion des eaux...) ;

- les outils contractuels, qui constituent un moyen souple de traduire concrètement des objectifs d'aménagement préalablement définis (les chartes intercommunales de développement et d'aménagement, les contrats de rivière, les contrats de plan, les conventions locales d'utilisation et/ou d'occupation des espaces riverains,...);

- les outils de planification, qui concernent l'organisation et la gestion des espaces (les documents d'urbanisme, les procédures réglementaires en relation avec l'occupation des zones inondables...);

- les outils de protection et de mise en valeur du patrimoine naturel qui sont en rapport avec :

- le milieu aquatique : notion de débit réservé, classement de cours d'eau, création de réserve de pêche..

- les espèces (flore-faune) et les espaces naturels: parcs naturels nationaux et régionaux, réserves naturelles, espaces naturels sensibles, zones naturelles d'intérêt écologique floristique et faunistique, zone de protection du patrimoine architectural urbain, sites classés et inscrits...

Les exigences d'une politique globale d'aménagement de rivière conduisent à ne négliger aucune piste. Au contraire, il s'agit d'envisager des combinaisons de procédures en fonction des objectifs poursuivis et des spécificités locales (tant sur le plan géographique, hydrologique et environnemental que social ou économique).

\section{Conclusion}

L'intervention croissante des collectivités territoriales et principalement des villes en matière d'aménagement et de gestion des cours d'eau ne doit pas faire oublier que c'est fréquemment l'improvisation et les opérations au coup par coup qui dominent encore. Il n'y a pas de véritables stratégies ni de volonté générale de réintégrer la rivière dans la ville.

De plus, il apparaît qu'il ne suffit pas d'infléchir les techniques d'étude et d'aménagement pour améliorer cette situation (même si la façon d'appréhender techniquement le milieu et de le traiter ultérieurement en ayant recours à tel ou tel matériau - conditionnent largement la perception que l'on peut avoir de cet aménagement). Il devient indis- pensable de mettre en œuvre des démarches spécifiques qui soient capables à la fois de :

- résoudre les problèmes ponctuels et quotidiens de l'eau en ville (assainissement, alimentation en eau potable, inondation...);

- percevoir des besoins incertains et évolutifs (pratiques sociales, demandes d'espaces publics, intentions d'usages...);

- convaincre et associer les partenaires indispensables (communes riveraines d'un même cours d'eau, usagers organisés ou non, services techniques de l'Etat...);

- rechercher un large consensus sur les schémas à entreprendre et les intégrer à des scénarios de développement à long terme.

Le niveau de prise de conscience des acteurs locaux et la volonté politique d'agir globalement sont les principaux facteurs à la base de toute la dynamique de mise en valeur des rivières.

\section{Références bibliographiques}

Barouch G. - La décision en miettes. Systèmes de pensée et d'action à l'auvre dans la gestion des milieux naturels,Paris, l'Harmatan, 1989, 237 p.

Denoziere P. - L'Etat et les eaux non domaniales, Paris, Lavoisier, $1985,354 \mathrm{p}$.

Dupuy G., KNAebel G. - Assainir la ville hier et aujourd'hui, Paris, Dunod, 1982, $92 \mathrm{p}$.

Gazzaniga J.L., Ourliac J.P. Le droit de l'eau, Paris, Litec, 1979, 247 p. + supplément à jour au $1^{\text {er }}$ septembre 1987,150 p.

G.R.A.I.E. - Les cahiers techniques pour l'aménagement et la gestion des cours d'eau, Paris, Min Environnement, Min Equipement (Plan Urbain); Lyon : Région Rhône-Alpes, Agence de l'eau R.M.C. I.N.G.U.L. ; 1991, 1000 p.

Goubert J.P. — La conquête de l'eau Paris, Robert Lafont, 1986, $296 \mathrm{p}$.

Guillerme A. - Les temps de l'eau. La cité, l'eau et les techniques, Seyssel, Champ-Vallon, 1983, 263 p.

Hubert G., Olivry D. - Des villes redécouvrent l'eau, 11 fiches de cas, Paris, E.N.P.C. (CERGRENE), Min Environnement; Lyon Agence de l'Eau R.M.C., 1987.

Hubert G., Olivry D. - Outils institutionnels, juridiques et financiers pour la valorisation des cours d'eau en milieu urbain, Paris, Min Equipement (Plan Urbain), 1989, $131 \mathrm{p}$.

HUBERT G. - Approche méthodologique pour la mise en valeur des rivières urbaines, Lyon, I.N.S.A., thèse de doctorat en Génie Civil et Sciences de la Conception, 1990, 486 p.

LE Moigne J.L. - La théorie du système général. Théorie de la modélisation,Paris, P.U.F., 1983, 258 p.

Metropolis L'eau urbaine Paris, revue Metropolis, n $92-93$, $1991,120 \mathrm{p}$.

MINISTÉRE DE L'ENVIRONNEMENT. - La gestion des rivières Paris, Min Environnement, les cahiers de l'environnement $n^{\circ} 1$, $1990,256 \mathrm{p}$. 